\title{
Inhibition of hIAPP Amyloid Aggregation and Pancreatic $\beta$-cell Toxicity by OH-terminated PAMAM Dendrimer
}

Esteban N. Gurzov, ${ }^{1,2}$ Bo Wang, ${ }^{3}$ Emily H. Pilkington, ${ }^{4}$ Pengyu Chen, ${ }^{5}$

Aleksandr Kakinen, ${ }^{4}$ William J. Stanley, ${ }^{1,2}$ Sara A. Litwak, ${ }^{1}$ Eric G. Hanssen, ${ }^{6}$

Thomas P. Davis, ${ }^{4,7^{*}}$ Feng Ding, ${ }^{3^{*}}$ and Pu Chun Ke $\mathrm{4}^{*}$

\begin{abstract}
${ }^{1}$ St Vincent's Institute of Medical Research, 9 Princes Street, Fitzroy, VIC 3065, Australia
${ }^{2}$ Department of Medicine, St. Vincent's Hospital, The University of Melbourne, Melbourne, Australia

${ }^{3}$ Department of Physics and Astronomy, Clemson University, Clemson, SC 29634, USA

${ }^{4}$ ARC Centre of Excellence in Convergent Bio-Nano Science and Technology, Monash Institute of

Pharmaceutical Sciences, Monash University, 381 Royal Parade, Parkville, VIC 3052, Australia

${ }^{5}$ Department of Mechanical Engineering, University of Michigan, Ann Arbor, MI, 28109, USA

${ }^{6}$ Bio21 Molecular Science and Biotechnology Institute, The University of Melbourne, Victoria 3010,

Australia

${ }^{7}$ Department of Chemistry, Warwick University, Gibbet Hill, Coventry, CV4 7AL, United Kingdom
\end{abstract}

*Address correspondence to:

Thomas P.Davis:thomas.p.davis@monash.edu, Feng Ding: fding@clemson.edu, and Pu Chun Ke:pu-chun.ke@monash.edu.

* E. Gurzov and B. Wang contributed equally to the presented work.

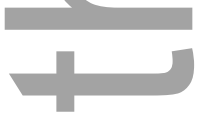

Supporting Information is available on the WWW under http://www.small-journal.com or

from the author.

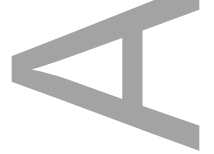

This is the author manuscript accepted for publication and has undergone full peer review but has not been through the copyediting, typesetting, pagination and proofreading process, which may lead to differences between this version and the Version of Record. Please cite this article as doi: 10.1002/smll.201502317.

This article is protected by copyright. All rights reserved. 
Keywords: hIAPP · amyloid fibrillation - protein aggregation - PAMAM-OH dendrimer · DMD simulations · high-throughput DLS · cytotoxicity

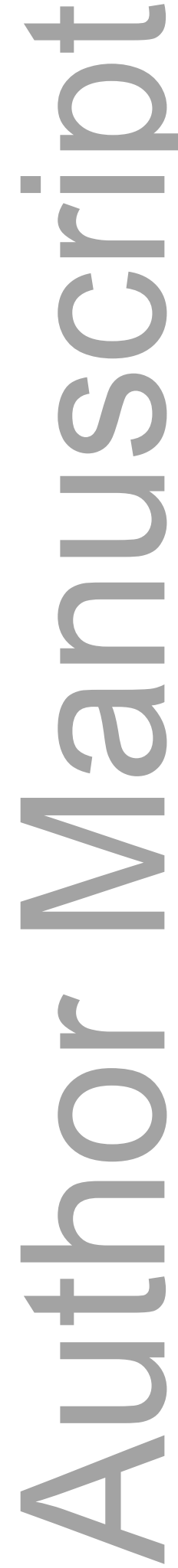

This article is protected by copyright. All rights reserved. 


\begin{abstract}
Human islet amyloid polypeptide (hIAPP, or amylin) forms amyloid deposits in the islets of Langerhans, a phenomenon that is associated with type-2 diabetes impacting millions of people worldwide. Accordingly, strategies against hIAPP aggregation are essential for the prevention and eventual treatment of the disease. Here we show that generation- $3 \mathrm{OH}-$ terminated poly(amidoamine) dendrimer, a polymeric nanoparticle, can effectively halt the aggregation of hIAPP and shut down hIAPP toxicity in pancreatic MIN6 and NIT-1 cells as well as in mouse islets. This finding was supported by our high-throughput dynamic light scattering experiment and thioflavin T assay, where the rapid evolution of hIAPP nucleation and elongation processes was halted by the addition of the dendrimer over time courses of up to $8 \mathrm{~h}$. Our rapid discrete molecular dynamics simulations further revealed that hIAPP residues bound strongly with the dendrimer near the c-terminal portion of the peptide, where the amyloidogenic sequence (residues 22-29) locates. Furthermore, simulations of hIAPP dimerization revealed that binding with the dendrimer significantly reduced formation of inter-peptide contacts and inter-peptide hydrogen bonds, thereby prohibiting peptide self-association and amyloidosis. This study points to a promising nanomedicinal strategy for combating type-2 diabetes and may have broader implications for targeting neurological disorders whose distinct hallmark is also amyloid fibrillation.
\end{abstract}

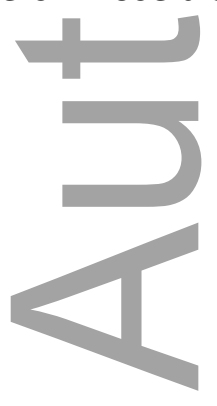

This article is protected by copyright. All rights reserved. 


\section{Introduction}

Islet amyloid polypeptide (IAPP, a.k.a. amylin) is a 37-residue peptide hormone, cosynthesized, co-stored and co-secreted with insulin by $\beta$-cells, which plays an important role in the glycemic control by slowing down gastric emptying. ${ }^{[1]}$ IAPP is one of the most amyloidogenic protein sequences known and amyloid deposits of IAPP are found in $~ 90 \%$ type-2 diabetes (T2D) patients. ${ }^{[2]}$ Despite the debate of whether islet amyloid is the cause or result of $\beta$-cell dysfunction, accumulating experimental evidences suggest that amyloid aggregation of IAPP contributes to the $\beta$-cell loss in T2D. ${ }^{[3,4]}$ IAPP aggregates - either insoluble amyloid fibrils or soluble oligomers - are found toxic to $\beta$-cells. ${ }^{[5,6]}$ IAPP variants from diabetes-prone primates and cats form aggregates readily in vitro, while those from diabetes-free rodents feature significantly weak aggregation propensities. ${ }^{[7]}$ A naturally occurring S20G mutation renders human IAPP (hIAPP) more aggregation-prone, while an Asian subpopulation carrying this mutation is subjected to early T2D onset. ${ }^{[8,9]}$ Moreover, transgenic mice expressing hIAPP start to develop diabetes. ${ }^{[10]}$ Therefore, as in many other amyloid diseases, ${ }^{[11,12]}$ inhibition of IAPP aggregation is an attractive therapeutic strategy to treat T2D. ${ }^{[13]}$

Stored inside $\beta$-cell granules at millimolar concentrations, hIAPP is natively inhibited due to interactions with other granule components, such as high concentrations of insulin, zinc, and proinsulin c-peptide. ${ }^{[14]}$ Disruption of the natively inhibiting cellular environment may contribute to the formation of amyloid aggregations in diabetes. ${ }^{[10]}$ In order to reduce the aggregation-induced cytotoxicity, anti-amyloid aggregation approaches - such as peptides, peptide-mimetics, ${ }^{[15-18]}$ and small molecules ${ }^{[19-26]}$ - have been exploited. Nonamyloidogenic sequence variants of hIAPP have been found to inhibit the fibril formation of 
hIAPP, ${ }^{[15,16]}$ and the inhibition efficacies can be improved by synthesizing peptide mimetics with conformational restraints. ${ }^{[17,18]}$ Several small polyphenol molecules, such as epigallocatechin gallate (EGCG), ${ }^{[19,20]}$ morin hydrate, ${ }^{[21]}$ myricetin, ${ }^{[22]}$ resveratrol, ${ }^{[23,24]}$ and curcumin, ${ }^{[25,26]}$ have also been found to inhibit the amyloid fibril formation of hIAPP. Compared to peptide inhibitors, small molecule inhibitors have greater therapeutics advantages. ${ }^{[27]}$ However, many of these IAPP aggregation-inhibiting polyphenols, e.g. myricetin, resveratrol ${ }^{[23,24] 23,24}$ and curcumin, have poor water solubility and therefore limited accessibility. On the other hand, recent advances in nanotechnology have opened up new possibilities for nanomedicine-based delivery of these anti-amyloid compounds.

PAMAM (polyamidoamine) dendrimers are a class of polymeric nanoparticles (NPS) with branching units iteratively emanating from a central core, adopting a unimolecular micelle structure in water with a hydrophobic interior and hydrophilic surface groups. ${ }^{[28,29]}$ The number of branching iterations corresponds to the "generation" of the dendrimer, which dictates the NP size and shape. Usually, PAMAM dendrimers have primary amines as the terminal groups, which are positively charged at neutral $\mathrm{pH}$ and may induce toxic effects, such as blood clot $^{[30]}$ and depolarization of the cell membrane. ${ }^{[31]}$ One advantage of PAMAM dendrimers is the straightforward modifications of their surface groups, including replacement of the dendrimer primary amines with hydroxyl groups to minimize the dendrimer toxicity and enhance its resemblance to polyphenols in surface properties, while maintaining the dendrimer high flexibility, small size and good water solubility.

PAMAM dendrimers can host a wide range of ligands, ${ }^{[32-35]}$ including several polyaromatic compounds, ${ }^{[36,37]}$ and have been used to encapsulate and deliver small hydrophobic pharmaceutical molecules for enhanced water solubility and biodistribution. ${ }^{[32]}$ This article is protected by copyright. All rights reserved. 
Poly(propyleneimine) (PPI) glycodendrimers have been employed for the prevention of Abeta aggregation. ${ }^{[38]}$ Phosphorous dendrimer, maltose-based glycodendrimer (mPPI), PPI vethyle and poly(ethyleneimine) hyperbranched polymer have demonstrated anti-prion activity. ${ }^{[39-}$ 44] Within the scope of T2D research, cationic PAMAM dendrimer has shown effectiveness in insulin aggregation inhibition. ${ }^{[45]}$ Generation-4 (G4) PAMAM dendrimer has shown the capacity to control blood glucose levels and mitigate hyperglycemia in diabetic rat models. ${ }^{[46]}$ In addition, a competitive binding scheme between fluorescently tagged Concanavalin-A and glycosylated PAMAM dendrimer has been demonstrated, which may be potentially developed into a minimally invasive glucose sensor for diabetics. ${ }^{[47]}$

In this work, we study the effect of generation-3 (G3) hydroxyl-terminated PAMAM (PAMAM-OH) On the amyloid aggregation of hIAPP and investigated their corresponding cytotoxicities using experimental biophysical characterizations combined with mechanistic in silico investigations and in vitro and ex vivo assays. We show that the presence of the dendrimer significantly and consistently reduced the cytotoxicity of hIAPP in MIN6 and NIT-1 cell lines as well as in mouse islets. This finding was supported by our high-throughput dynamic light scattering (DLS) experiment and thioflavin T (ThT) spectroscopic assay, where the aggregation of hIAPP was halted by the addition of the dendrimer over time courses of up to $8 \mathrm{~h}$. Our discrete molecular dynamics (DMD) simulations revealed that the dendrimer had a strong binding to hIAPP monomers, especially the amyloidogenic sequence (residues 22-29). ${ }^{[48]}$ Moreover, simulations of hIAPP dimerization revealed that binding with the PAMAM-OH dendrimer significantly reduced the formation of inter-peptide contacts and inter-peptide hydrogen bonds, thereby prohibiting peptide self-association and amyloid formation. Overall, our multi-scale and multi-disciplinary approaches converge on the 
discovery that polymeric PAMAM-OH dendrimers may act as a promising T2D nanomedicine, and may find additional use in dealing with neurological disorders that are also characterized by amyloid fibrillation.

\section{Results and Discussion}

\subsection{Experimental evidences of hIAPP fibrillation inhibition by G3 PAMAM-OH dendrimer}

The zeta potentials of hIAPP and PAMAM-OH dendrimer were first determined to be +4.71 $( \pm 0.39) \mathrm{mV}$ and $-10.5( \pm 1.51) \mathrm{mV}$ in Milli-Q water at room temperature. In comparison, hIAPP-dendrimer mixture at a 1:1 molar ratio rendered a zeta potential of $-4.85( \pm 0.38) \mathrm{mV}$. TEM imaging showed the occurrence of abundant amyloid fibrils after overnight incubation of hIAPP at $0.1 \mathrm{mg} / \mathrm{mL}(26 \mu \mathrm{M})$, which were usually ranged over $1 \mu \mathrm{m}$ in length (Figure 1A) and $7 \sim 14 \mathrm{~nm}$ in width. This is consistent with the literature ${ }^{[49]}$ and the understanding that hIAPP at micromolar concentrations is prone to fibrillation through molecular assembly. ${ }^{[50]}$ In the sample where hIAPP and dendrimer were mixed with equal volumes, short (tens of nm) fibrils were occasionally spotted and associated with small spherical objects of less than 10 $\mathrm{nm}$ in size (Figures 1B, C). These spherical objects were prevalent regardless of the presence of fibrils (Figures 1B-D), and are inferred to be hIAPP-dendrimer clusters as they were absent in either the dendrimer (Figure S1) or the hIAPP control images (Figure 1A). Colocalization of such spherical particles with fibrils in Figures $1 \mathrm{~B} \& \mathrm{C}$ further vindicates binding between the dendrimer and the peptide species. Similar spherical nanostructures were reported for G5 PAMAM dendrimers complexing with linear zwitterionic chitosan. ${ }^{[51]}$ This 
result suggests that PAMAM-OH dendrimer could act as an inhibitor for hIAPP polymerization in the aqueous phase.

Amyloid aggregation of hIAPP was evident from the high-throughput DLS measurement (Figures 1E, F). At the initial time point (Figure 1F, 0 min), hIAPP displayed a single major peak of hydrodynamic diameter (by mass percentage) centered at $2.2 \mathrm{~nm}$ $( \pm 0.7)$, corresponding to the size of a peptide monomer. As time progressed, the single peak of the peptide evolved into more irregular and broader distributions, characterized by double or multiple peaks at significantly larger sizes of tens of nanometers to micrometers. Specifically, two prominent peaks were seen for hIAPP aggregates at the time points of 45th min and 130th min, while three major peaks were recorded at the time point of 360 th min, centered at $56.5 \mathrm{~nm}, 767.8 \mathrm{~nm}$ and $4,590.8 \mathrm{~nm}$, respectively. At the last registered time point of 470 th $\min$, two major peaks occurred at $58.1 \mathrm{~nm}$ and 3,583.4 $\mathrm{nm}$ (Figure 1E). The dendrimer controls, in comparison with the hIAPP samples, displayed little variations in hydrodynamic size over time, at a highly monodisperse value of $2.2 \mathrm{~nm}( \pm 0.1)$ (Figure $1 \mathrm{~F}$ inset). Here the size of G3 PAMAM was averaged over 20 data points, with each trace ranging between 2-4 $\mathrm{nm}$ in Figure $1 \mathrm{~F}$ inset. These are reasonable hydrodynamic sizes of the G3 dendrimer and hIAPP monomers, especially considering the instrument resolution $(0.5$ $\mathrm{nm})$ and ultrasmall scattering sample volumes $(20 \mu \mathrm{L})$ of the high-throughput DLS technique. A direct comparison between the TEM and DLS sizes of the dendrimer or hIAPP was not feasible due to the different samples conditions (dehydrated vs. hydrated state) and different working principles and sensitivities of the two techniques. Consistently with the TEM imaging, when the peptide and the dendrimer were mixed together at a molar ratio of 6:7, the process of amyloid aggregation of hIAPP was largely stalled (Figure 1F), yielding a single 
major peak at $2.6 \mathrm{~nm}( \pm 0.3)$ in hydrodynamic size. In consideration of the $6^{\text {th }}$ power size dependence of DLS we exclude the presence of traceable hIAPP fibrils in the sample Comp

mixture. Comparable results were seen for hIAPP-dendrimer of two other molar ratios of 12:7 and 3:7 (data not shown), indicating effectiveness of using PAMAM-OH dendrimer for the inhibition of hIAPP aggregation.

Thioflavin T (ThT) is a commonly used assay for the visualization and quantification of amyloid fibrillation induced by protein misfolding and aggregation. ${ }^{[52]}$ Specifically, binding of ThT dye to cross-beta architecture induces an enhanced fluorescence intensity, sometimes accompanied by a redshift in the peak fluorescence wavelength. Changes in the ThT fluorescence intensities are used to indicate the amount of amyloid aggregates. Figure 2 shows the time-course data of hIAPP with the empirical sigmoidal fitting (dashed lines). Upon excitation of the ThT-stained samples at $440 \mathrm{~nm}$, the control hIAPP displayed the highest change in fluorescence intensity indicating a rapid amyloid formation of pure hIAPP over time. Addition of PAMAM-OH dendrimer resulted in a significant decrease in the fluorescence intensity signifying inhibited amyloid aggregation. Specifically, the maximal inhibition of aggregation was observed at the highest dendrimer to hIAPP molar ratio (10:1) with the fluorescence signal being 2.3 folds less than it was for the control hIAPP at the plateaus. ThT at $25 \mu \mathrm{M}$, which is significantly above its critical micelle concentration of 4 $\mu \mathrm{M},{ }^{[53]}$ assumed micelles in the sample mixtures. Compared to $\mathrm{H}$-bonding and electrostatic interactions that are favorable between ThT micelles and amyloids, ${ }^{[53]}$ the interaction between ThT micelles and PAMAM-OH dendrimer could only play a minimal role judging from the absence of complete fluorescence quenching at high dendrimer concentrations $(10 \sim 100 \mu \mathrm{M})$. 
Fitted with the empirical sigmoidal function (Experimental Section), the amount of amyloid aggregates corresponding to the maximum ThT fluorescence intensity can be obtained as a function of relative dendrimer/hIAPP ratio (Figure 2 inset). At relatively low dendrimer/hIAPP ratios $(\leq 0.2)$, a linear decrease of total aggregates with increasing dendrimer/hIAPP ratio was observed. Such linear dependence suggests an inhibition scenario, where the PAMAM-OH dendrimer inhibited hIAPP amyloid aggregation by sequestering hIAPP monomers. The linear decrease of amyloid aggregations slowed down at higher dendrimer/hIAPP ratios, likely a result of the self-association of dendrimers via their hydrophobic interiors exposed through constant thermal fluctuations. Nevertheless, the total amount of amyloid aggregates continued to decrease monotonically with increased dendrimer, highlighting strong amyloid inhibition by the polymeric NPs. Specifically, the time $t_{0}$ taken for the reaction to reach the half intensity maximum was delayed from 170 min for the hIAPP control to $191 \mathrm{~min}$ at the highest dendrimer/hIAPP molar ratio of 10:1, while the apparent rate constant of fibrillation $k$ was reduced from $0.037 \mathrm{~min}^{-1}$ to $0.026 \mathrm{~min}^{-}$ 1 .

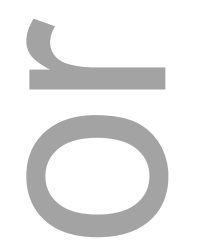

\subsection{Molecular details of hIAPP fibrillation inhibition by g3 PAMAM-OH dendrimer}

To understand the mechanisms of hIAPP aggregation inhibition by PAMAM-OH, atomistic discrete molecular dynamics (DMD) simulations were conducted (Experimental Section). We studied the binding of generation-3 PAMAM-OH dendrimer with a hIAPP monomer as well as the effect of PAMAM-OH dendrimer on hIAPP dimerization, an important step toward amyloid aggregation. ${ }^{[48,54]}$ Since the binding of multiple macromolecules is highly stochastic (e.g., Figure S2), we performed ten independent simulations for each molecular system to 
ensure sufficient sampling. Each simulation started with different initial conditions, including randomized velocities, different inter-molecular distances and orientations. With each simulation $\sim 100 \mathrm{~ns}$ long, we obtained an accumulative $1 \mu$ s simulation for each studied molecular system.

\subsubsection{Dendrimer binds to the amyloidogenic sequence of individual hIAPP}

Simulation on dendrimer binding to individual hIAPP was initialized by assigning a hIAPP monomer near a PAMAM-OH dendrimer with random orientations. In order to examine the possible conformational changes of the hIAPP while interacting with the dendrimer, we also simulated the hIAPP monomer itself as a control. We calculated the contact frequency (Figure 3A) and number of atomic contacts (Figure 3B) between each individual hIAPP residue and the dendrimer. Hot-spot residues with peaks in the number of contact and relative high binding frequency were observed, which implied strong inter-molecular binding. Interestingly, many of hot-spot residues located near the c-terminal, corresponding to the amyloidogenic residues (residues 22-29). ${ }^{[48]}$ Examination of the molecular complex in simulations (e.g., a snapshot structure in Figure 3C) suggests that the hIAPP was embraced by the flexible dendrimer, especially near the amyloidogenic region. A comparison of the peptide secondary structures between hIAPP alone and in the presence of dendrimer (as illustrated in Figure 3D) indicates that no significant difference was induced, which can be explained by the flexible nature of low-generation dendrimers. ${ }^{[55]}$

\subsubsection{Binding with dendrimer inhibited inter-hIAPP interactions}

We further simulated one dendrimer with two separated hIAPP monomers, where each hIAPP monomer was positioned away from the PAMAM-OH dendrimer with random intermolecular distances and orientations. For comparison, we performed simulations of two hIAPP monomers. We first calculated the average number of hIAPP peptides bound to the

This article is protected by copyright. All rights reserved. 
dendrimer as a function of time (Figure 4A). Despite the initial fluctuations, the number was close to $\sim 1.2$ near the end of the simulations, consistent with the DLS observations of peptide aggregation inhibition at hIAPP/dendrimer molar ratios of 12:7, 6:7 and 3:7. The effect of the dendrimer on inter-hIAPP interactions was evaluated by computing the histograms of the number of inter-peptide contacts (Figure 4B) and hydrogen bonds (Figure 4C). In the presence of the dendrimer, the probability of observing a higher number of inter-peptide contacts, $P_{n c}$, was reduced. Similarly, the number of inter-peptide hydrogen bonds, $N_{h b o n d}$, was also significantly reduced. Especially, in the presence of the dendrimer, the exponential decrease of the probability of observing a given number of inter-chain hydrogen, $P_{h b o n d}$, with increased $N_{\text {hbond }}$ suggests no formation of stable $\beta$ sheet like structures between the two peptides. In contrast, peaks (shoulders) of the $P_{h b o n d}$ profile in the hIAPP dimer simulations corresponded to the formation of stable (meta-stable) $\beta$ sheets.

To further characterize the structural properties of hIAPP dimers, we computed the inter-monomer contact frequency for all residue pairs with and without the dendrimer. In the case of hIAPP dimer alone, high frequency areas were mostly along the diagonal near the amyloidogenic regions (Figure 5A), consistent with the formation of a parallel $\beta$ sheets conformation between the amyloidogenic regions (Figure 5B) as observed previously. ${ }^{[48]}$ In the presence of the dendrimer, we observed a more scattered pattern (Figure 5C). The relatively strong inter-peptide binding occurred in the hydrophobic region near the Nterminal (residues $15-17, \mathrm{~F}^{15} \mathrm{LV}^{17}$ ) as illustrated by a snapshot structure with the dendrimer bound to both hIAPP peptides (Figure 5D), while the amyloidogenic region of one of the peptides (corresponding to the yellow region in Figure 5D) were protected by binding with the dendrimer. It should be noted that in the aqueous phase, unlike in silico, there is little control for hIAPP to assume the monomer or dimer state due to its rapid fibrillation kinetics. In this sense DMD simulations are advantageous over experiments in revealing the 
mechanisms of hIAPP-dendrimer interaction. Extrapolating the current observation from hIAPP dimer to oligomers and fibrils, we conclude that PAMAM-OH dendrimer inhibited amyloid aggregation of hIAPP by binding to and encapsulating the amyloidogenic sequences, thus preventing their self-association and formation of inter-peptide hydrogen bonds toward amyloid fibrillation.

\subsection{PAMAM-OH dendrimer protects B-cells from hIAPP-induced cell death}

Next, we determined whether inactivation of hIAPP aggregation by PAMAM-OH can prevent the peptide toxicity in pancreatic $\beta$-cells. For this purpose, the mouse $\beta$-cell lines MIN6 and NIT-1 were treated with $10 \mu \mathrm{M}$ hIAPP, $6 \mu \mathrm{M}$ PAMAM-OH, or combination of both. After $24 \mathrm{~h}$ treatment, the cells were exposed to the DNA binding dyes Hoechst 33342 and propidium iodide. Hoechst 33342 freely diffuses and enters cells with intact or damaged membranes, staining DNA blue. Propidium iodide, a highly polar dye, is impermeable to cells with preserved membranes and only stains DNA of death cells as red. Viable cells were identified by their intact nuclei with blue fluorescence, whereas cell death was quantified by blue-red fluorescence or by fragmented blue nuclei. ${ }^{[56]}$

As a control we first treated NIT-1 cells with PAMAM-OH $(6 \mu \mathrm{M})$ for $24 \mathrm{~h}$, stained them with Hoechst-33342, and performed immunofluorescence and light microcopy. The dendrimers did not interfere with nuclear DNA staining, as clearly shown in Figure 6. We then observed that culture of the mouse $\beta$-cell lines with $10 \mu \mathrm{M}$ hIAPP for $24 \mathrm{~h}$ resulted in 50-90\% cell death (Figures 7A, B), as previously reported. ${ }^{[57]}$ However, pre-treatment of hIAPP with $36 \mu \mathrm{M}$ PAMAM-OH inhibited fibril formation and prevented hIAPP-induced cell death (Figures 7A, B). Consistent results were observed for pre-treatment of hIAPP with 
equal molar concentration of PAMAM-OH (both at $10 \mu \mathrm{M}$ ) in NIT-1 cells (Figure S3). Importantly, we confirmed this result in primary cells. Uniformly sized mouse islets from mice

C57BL/6 mice were handpicked into 1-cm Petri dishes containing $1.1 \mathrm{~mL}$ of $10 \mu \mathrm{M}$ hIAPP, 6 $\mu \mathrm{M}$ PAMAM-OH or combination of both, and cultured for $48 \mathrm{~h}$. At the end of the culture period, islets were dispersed with trypsin, and resuspended in $250 \mu \mathrm{L}$ of hypotonic buffer containing $50 \mu \mathrm{g} / \mathrm{mL}$ propidium iodide, which stained nuclear DNA. The cells were analyzed by fluorescence-activated cell sorter (FACS), and cell death was identified by their subdiploid DNA content as previously described. ${ }^{[58]}$ Dispersed mouse islets incubated with hIAPP for 48 h showed $25 \%$ viability, whereas pre-incubation with PAMAM-OH reduced hIAPP-mediated cell death by more than $50 \%$ (Figure $7 \mathrm{C}$ ). These in vitro and ex vivo assays consistently point to the direction that PAMAM-OH dendrimer is an effective inhibitor of hIAPP-induced cytotoxicity.

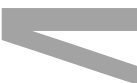

A number of mechanisms have been proposed in the literature concerning hIAPPinduced cytotoxicity: disruption of membrane fluidity and the subsequent intracellular hemostasis, generation of reactive oxygen species (ROS) which results in cell death, and apoptosis. ${ }^{[59]}$ Due to its hydrophilicity and weakly negative charge, PAMAM-OH dendrimer alone is not expected to show notable cell uptake. On the other hand, although hIAPP is weakly positively charged $(4.71 \mathrm{mV})$, binding of hIAPP with PAMAM-OH dendrimer rendered a slightly negative zeta potential of $-4.85 \mathrm{mV}$, thereby reducing propensity of the peptide for negatively charged cell membranes to discourage membrane adsorption and cell uptake. Furthermore, encapsulation of hIAPP monomers and their small structural derivatives (i.e., dimers and oligomers) by G3 PAMAM-OH dendrimer prevented the peptides from evolving 
into hydrophobic fibrils, further minimizing hIAPP perturbation to cell membranes and cell uptake.

Using the $\mathrm{CM}-\mathrm{H}_{2}$ DCFDA assay, Feliu et al. showed that G4 PAMAM-OH dendrimers at concentrations lower than $50 \mu \mathrm{M}$ elicited no ROS in primary human bronchial epithelial cells. ${ }^{[60]}$ It is conceivable that G3 PAMAM-OH dendrimer used in our study, due to its much reduced numbers of surface groups ( 32 per G3 vs. 64 per G4 dendrimer) and branches for a given molar concentration, would be comparably or less active in ROS production. Furthermore, binding with the dendrimer could also reduce ROS production by the peptides and their aggregates, as electron-donor peptide residues could be partially buried inside the dendrimer to become less accessible to oxygens and free radicals in the cellular environment. Specifically, all the oxidation-prone residues ${ }^{[61]}$ of hIAPP, including Phe15, His18, Phe23 and Tyr37, showed strong binding with the dendrimer (Figures 3A, B) and thus were buried inside the dendrimer upon interactions (Figure 3C). A similar phenomenon was observed for the case of hIAPP dimer interacting with the dendrimer (Figures S4, 5D).

Although limited data is available in literature with regard to the apoptotic potential of dendrimers, $\mathrm{G} 3$ PAMAM-OH dendrimers at concentrations up to $500 \mu \mathrm{M}$ - one to two orders of magnitude higher than those employed in our cellular studies - have been shown none apoptotic in MCF7 and MDA-MB-231 breast cancer cells. ${ }^{[62]}$ In short of above analysis, it is plausible that the presence of G3 PAMAM-OH dendrimer physically or physicochemically screened and mitigated the cytotoxicity of hIAPP.

\section{Conclusion}

This article is protected by copyright. All rights reserved. 
We have demonstrated the remarkable effect of G3 PAMAM-OH dendrimer on the inhibition of hIAPP aggregation. While hIAPP of micromolar concentrations underwent rapid nucleation and elongation in aqueous (Figures $1 \mathrm{~A}, \mathrm{C}$; Figure 2), the presence of the dendrimer effectively halted hIAPP polymerization (Figures 1B, D; Figure 2). The molecular details of such peptide-dendrimer interactions and their induced inhibition of peptide aggregation were further revealed by DMD simulations, where the dendrimer bound to the amyloidogenic regions of hIAPP monomers and impaired the formation of inter-peptide hydrogen bonds and hydrophobic interactions between hIAPP dimers toward amyloid fibrillation (Figures 3-5).

When hIAPP-dendrimer were introduced to MIN6 and NIT-1 cells and mouse islets, all cell lines and primary cells consistently exhibited significantly reduced cytotoxicity compared with those exposed to hIAPP alone (Figure 7). This phenomenon was attributed to the decreased zeta potential (and hence declined affinity) and stalled fibrillation (and hence reduced perturbation) of the peptide for cell membranes upon dendrimer binding, as well as reduced cellular exposure of the oxidation-prone peptide residues that were buried in the dendrimer. Taken together, G3 PAMAM-OH dendrimer not only effectively hindered hIAPP aggregation in aqueous and in silico, but also significantly mitigated the toxic effects of the peptides and their aggregates in vitro and ex vivo. In practice, the high cost of G3 PAMAM-OH dendrimer may be circumvented by the use of its analogue that is comparable in both structural and physicochemical properties, the G3 hyperbranched bis-MPA polyester-32-hydroxyl polymer. This research offers a novel strategy for targeting T2D as well as neurological disorders, and opens the door to the screening and development of other classes of NPs and nanomedicines for amyloid inhibition. 


\section{Experimental Section}

Sample preparations: Human islet amyloid polypeptide (hIAPP) (KCNTATCATQRLANFLVHSSNNFGAILSSTNVGSNTY; disulfide bridge: 2-7; MW: 3,906) was obtained from AnaSpec in lyophilized powder form. The hIAPP was weighed on a Cubis MSE balance (Sartorius, $0.01 \mathrm{mg}$ resolution) and dissolved in Milli-Q water to form a $0.25 \mathrm{mg} / \mathrm{mL}(64 \mu \mathrm{M})$ stock solution immediately prior to the measurements. Generation-3 (G3) PAMAM-OH dendrimer (stock concentration: 6.25\% in water; MW: 6,941) was acquired from Dendritech (Midland, Michigan) and was diluted in Milli-Q water to 0.78 $\mathrm{mg} / \mathrm{mL}(112 \mu \mathrm{M})$. Each PAMAM-OH dendrimer constituted an ethylenediamine core and 32 amidoethanol surface groups.

Zeta potential: The zeta potentials of hIAPP $(64 \mu \mathrm{M})$, PAMAM-OH dendrimer $(112 \mu \mathrm{M})$ and hIAPP-dendrimer (1:1 molar ratio) in Milli-Q water were determined using a DLS device (Zetasizer Nano S90, Malvern Instruments) at room temperature.

TEM: Copper grids (300-mesh, carbon coated) were glow-discharged in nitrogen to promote hydrophilicity. Aqueous hIAPP $(0.1 \mathrm{mg} / \mathrm{mL}$ or $26 \mu \mathrm{M})$, PAMAM-OH dendrimer $(0.31 \mathrm{mg} / \mathrm{mL}$ or $45 \mu \mathrm{M}$ ), and a hIAPP-dendrimer mixture (of equal volume, or 26:45 molar ratio), all incubated overnight at $4^{\circ} \mathrm{C}$, was pipetted onto the grids and allowed $20 \mathrm{~s}$ of adsorption. Excess samples were then drawn off using filter paper and the grids washed using Milli-Q water. Staining was undertaken using $2 \%$ uranyl acetate for $20 \mathrm{~s}$, with excess stain drawn off as previously described. The grids were dried under heated airflow for 20-30 s or as needed. The samples were then examined using a Tecnai G2 F30 Transmission Electron Microscope 
(FEI, Eindhoven, The Netherlands), operating at a voltage of $300 \mathrm{kV}$. Images were recorded using Gatan UltraScan 1000 (2k×2k) CCD camera (Gatan, California, USA).

High-throughput DLS: The hydrodynamic diameters of the hIAPP (stock: $64 \mu \mathrm{M}$ ), PAMAM-OH dendrimer (stock: $112 \mu \mathrm{M}$ ) and hIAPP-dendrimer mixtures were measured using an automated, high-throughput DLS device (DynaPro Plate Reader, Wyatt; resolution: $0.5 \mathrm{~nm}$ ) and black 384-well plates (Thermo Fisher). ${ }^{[63]}$ For each hIAPP-dendrimer mixture, the volume of the hIAPP was fixed at $6 \mu \mathrm{L}$ while the volume of the PAMAM-OH dendrimer was increased from $2 \mu \mathrm{L}$ to $4 \mu \mathrm{L}$ and $8 \mu \mathrm{L}$, rendering a final hIAPP concentration of $19 \mu \mathrm{M}$ and a hIAPP/dendrimer molar ratio of 12:7, 6:7 and 3:7, respectively. For the controls both the hIAPP and the dendrimer were fixed at $6 \mu \mathrm{L}$. The volume of each sample well was topped up to $20 \mu \mathrm{L}$ by Milli-Q water. Prior to the DLS measurement the samples were spun for 1 $\min$ at 1,000 rpm/164 RCF (Centrifuge 5804, Eppendorf) to ensure uniform mixing. An optical module collected data at 20 acquisitions per sample well at room temperature, which was then automatically processed and displayed using the Dynamics 7.1.7 software. To ensure repeatability and statistics each sample condition was measured with quadruplicate. The DLS experiment ran for $470 \mathrm{~min}$ in total.

ThT assay: To study the binding kinetics of hIAPP amyloid fibrils formation, the hIAPP stock was diluted to a final concentration of $10 \mu \mathrm{M}$ in $100 \mathrm{mM}$ Tris- $\mathrm{HCL}(\mathrm{pH}=7.5), 100 \mathrm{mM} \mathrm{NaCl}$ buffer and mixed with $25 \mu \mathrm{M}$ ThT (Sigma) and varied concentrations of PAMAM-OH dendrimer. Then $100 \mu \mathrm{L}$ of the each prepared samples was added to a 96 well plate and changes in ThT fluorescence intensity in the control and the hIAPP-dendrimer mixtures were recorded every 5 min over $8 \mathrm{~h}$ at a constant temperature of $25^{\circ} \mathrm{C}$ using a BioTek Synergy H1 Hybrid Reader (Excitation: $440 \mathrm{~nm}$; Emission: $485 \mathrm{~nm}$ ). The time course data were fitted using an empirical equation: 


$$
I(t)=I_{0}+\left(I_{\max }-I_{0}\right) /\left\{1+\exp \left[-k\left(t-t_{0}\right)\right]\right\}
$$

Where $I_{0}$ and $I_{\max }$ are the initial and maximum fluorescence intensity, $k$ is the apparent rate constant of fibrillation, and $t_{0}$ is the time required to reach a half of the maximum intensity changed.

Discrete molecular dynamics (DMD): $\mathrm{DMD}$ is a special type of molecular dynamics (MD) technique where inter-atomic interactions are modeled by step-wise potential functions instead of continuous potentials. Without frequent calculations of forces at a short time step ( femtosecond), the computational efficiency of DMD is increased. The detailed description of the DMD algorithm can be found elsewhere. ${ }^{[64,65]}$ By following the same physical laws and with discretized inter-atomic potential functions mimicking molecular mechanics-based force field, ${ }^{[6,67]}$ the dynamics in DMD is equivalent to continuous potential MD at timescales larger than picoseconds with differences mainly at short timescales within the sub-picosecond range (i.e., the average time step between two consecutive inter-atomic collisions where a potential energy step is encountered). The predictive power of the atomistic DMD simulations has been demonstrated by observing $a b$ initio folding (i.e., without the tertiary structure information in the force field) of a set of small proteins, ${ }^{[66]}$ recapitulating the relative folding rates of different proteins, ${ }^{[68]}$ and capturing the coupled conformational dynamics of large proteins that have been used to engineer allosterically regulated kinases. ${ }^{[69]}$ Recently, we also showed that DMD simulations could recapitulate the experimentally-determined radius of gyration $(R g)$ of PAMAM dendrimers of different generations. In addition to the $R g$ values, the atomic distributions and the corresponding dynamics of PAMAM dendrimers in DMD simulations were consistent with all-atom MD simulations. ${ }^{[37]}$ Therefore, we applied DMD simulations to study the binding of PAMAM This article is protected by copyright. All rights reserved. 
dendrimers with hIAPP peptides.

In our simulations, all molecules were represented by the united-atom model, where all heavy atoms and polar hydrogen atoms were explicitly modeled. We used implicit solvent in the simulations. Inter-atomic interactions considered included van der Waals (VDW), solvation, electrostatic interaction and hydrogen bond. The solvation energy was estimated using the Lazaridis-Karplus implicit solvent model, EEF1. ${ }^{[70]}$ The distance and angular dependent hydrogen bond interaction was modeled using a reaction-like algorithm. ${ }^{[71]}$ We used the Debye-Hückel approximation to model the screened electrostatic interactions between charged atoms. The Debye length was approximately $1 \mathrm{~nm}$ by assuming water relative permittivity of 80 and a monovalent electrolyte concentration of $0.1 \mathrm{M}$.

The coordinates of hIAPP were obtained from the protein data bank (PDB code 2L86). ${ }^{[72]}$ G3 PAMAM-OH was generated as described previously. ${ }^{[37]}$ Basic and acidic amino acids of the hIAPP were assigned charges according to their titration states at physiological $\mathrm{pH}=7.4$. The Arg and Lys residues were assigned +1 , the Asp and Glu were assigned -1 , while the His was neutral. Tertiary amines in the PAMAM-OH dendrimer were uncharged. The net charges of the molecular systems were maintained at zero by adding offsetting charges $\left(\mathrm{Cl}^{-}\right)$ to account for possible counterion condensation. ${ }^{[73]}$ In DMD simulations, temperature had the unit of $\mathrm{kcal} /\left(\mathrm{mol} \bullet k_{B}\right)$, where $k_{B}$ is the Boltzmann constant. Our simulations were conducted at $0.60 \mathrm{kcal} /\left(\mathrm{mol} \bullet \mathrm{k}_{B}\right)$, corresponding to room temperature $\mathrm{T}=300 \mathrm{~K}$, with a scaling factor of $\sim 500$. The Anderson's thermostat was used to maintain constant temperature. 
We simulated one dendrimer interacting with one hIAPP monomer and also with two hIAPP monomers. In each case, we initialized the molecular system by assigning hIAPP monomers away from the dendrimer with random orientations. We also performed simulations of a single hIAPP monomer and two hIAPP monomers as the controls. To ensure sufficient sampling and avoid potential bias of initial conditions, we performed ten independent simulations for each molecular system starting with different initial configurations, including randomized velocities, different inter-molecular distances and orientations. For each independent simulation, energy minimization was first carried out for one thousand time steps (approximately 0.05 ns with 1 DMD time step corresponding to $\sim 50 \mathrm{fs}$ ), followed by production simulations. Each production run lasted two million steps (approximately 100 ns), corresponding to an average of approximately 144 CPU hours.

A contact between a residue of hIAPP and PAMAM-OH dendrimer (or two residues of hIAPP) was defined with at least one heavy-atom contact (A $5 \AA$ cutoff was used). We computed the content of secondary structures - helix, strand turn and coil - using the backbone dihedral angles. ${ }^{[74,75]}$ All the quantitative calculations, including the mean and standard deviation of contacts and secondary structure contents, were obtained from the latter half of production runs where the molecular systems were found in equilibrium. For each simulation, we used 10,000 snapshots evenly distributed along the final 50 ns for analysis.

Cell culture and viability: C57BL/6 mice were maintained at St. Vincent's Institute and experiments were approved by the institutional animal ethics committee. Mouse islets were isolated and cultured as described previously. ${ }^{[76]}$ Islets were dispersed into single cells with trypsin and DNA fragmentation was analyzed by staining with propidium iodide. ${ }^{[77]}$ The 
insulin producing MIN6 and NIT-1 cell lines were cultured in DMEM (Invitrogen, UK) supplemented with $10 \%$ fetal calf serum. The percentage cell death of MIN6 and NIT-1 cells was determined in at least 600 cells per experimental condition by inverted fluorescence microscopy after staining with the DNA dyes Hoechst-33342 $(10 \mu \mathrm{g} / \mathrm{mL})$ and propidium iodide $(5 \mu \mathrm{g} / \mathrm{mL})$. The cells and islets were cultured in Opti-MEM (Life Technologies) for treatment with hIAPP $(10 \mu \mathrm{M})$, PAMAM-OH $(6 \mu \mathrm{M})$ or $10 \mu \mathrm{M}$ hIAPP pre-treated for $16 \mathrm{~h}$ with $36 \mu$ M PAMAM-OH. hIAPP and PAMAM-OH concentrations were selected according to published data and our own dose response ${ }^{[78]}$. The cells were treated for $24 \mathrm{~h}$ and the islets for $48 \mathrm{~h}$ before the viability assessment.

Statistical analysis: Data are represented as means \pm SEM. Given the paired nature of the experimental design, comparisons between treated groups were made by analysis of variance (ANOVA). A p value $<0.05$ was considered statistically significant.

ACKNOWLEDGMENT This work was supported by ARC Project No. CE140100036 (Davis), NSF CBET-1232724 (Ke and Ding), NIH R15ES022766-01A1 (Ding), and NHMRC Project Grant APP1071350 (Gurzov). Davis is thankful for the award of an Australian Laureate Fellowship from the ARC. Gurzov is supported by a Juvenile Diabetes Research Foundation (JDRF) fellowship. The St Vincent's Institute receives support from the Operational Infrastructure Support Scheme of the Government of Victoria. The authors acknowledge Dr. Shane Seabrook for assistance with the DLS measurement. All simulations were performed on the Palmetto high performance cluster at Clemson University CCIT. 


\section{References}

[1] O.Schmitz, B. Brock, J. Rungby, Diabetes 2004, 53, S233.

[2] S. E. Kahn, S. Andrikopoulos, C. B. Verchere, Diabetes 1999, 48, 241.

[3] J. W. M. Höppener, B. Ahrén, C. J. M. Lips, N. Engl. J. Med. 2000, 343, 411.

[4] J. W. M. Höppener, C. J. M. Lips, Int. J. Biochem. Cell Biol. 2006, 38, 726.

[5] S. Zraika, R. L. Hull, C. B. Verchere, A. Clark, K. J. Potter, P. E. Fraser, D. P. Raleigh, S. E. Kahn, Diabetologia 2010, 53, 1046.

[6] R. A. Ritzel, J. J. Meier, C.-Y. Lin, J. D. Veldhuis, P. C. Butler, Diabetes 2007, 56, 65.

[7] S. Chakraborty, B. Chatterjee, S. Basu, Biophys. Chem. 2012, 168-169, 1.

[8] S. Seino, Study Group of Comprehensive Analysis of Genetic Factors in Diabetes Mellitus, Diabetologia 2001, 44, 906.

[9] S. Sakagashira, H. J. Hiddinga, K. Tateishi, T. Sanke, T. Hanabusa, K. Nanjo, N. L. Eberhardt, Am. J. Pathol. 2000, 157, 2101.

[10] P. Westermark, A. Andersson, G. T. Westermark, Physiol. Rev. 2011, 91, 795.

[11] F. E. Cohen, J.W. Kelly, Nature 2003, 426, 905.

[12] T. Klabunde, H. M. Petrassi, V. B. Oza, P. Raman, J. W. Kelly, J. C. Sacchettini, Nat. Struct. Biol. 2000, 7, 312 .

[13] P. Cao, A. Abedini, D. P. Raleigh, Curr. Opin. Struct. Biol. 2013, 23, 82.

[14] P. Westermark, Z.-C. Li, G. T. Westermark, A. Leckström, D. F. Steiner, FEBS Lett. 1996, 379, 203.

[15] A. Abedini, F.Meng, D. P. Raleigh, J. Am. Chem. Soc. 2007, 129, 11300.

[16] D. Sellin, L.-M. Yan, A. Kapurniotu, R. Winter, Biophys. Chem. 2010, 150, 73.

[17] L.-M. Yan, A. Velkova, M. Tatarek-Nossol, E. Andreetto, A. Kapurniotu, Angew. Chem. Int. Ed. 2007, 46, 1246.

[18] L.-M. Yan, M. Tatarek-Nossol, A. Velkova, A. Kazantzis, A. Kapurniotu, Proc. Natl. Acad. Sci. U. S. A. 2006, 103, 2046.

[19] F. Meng, A. Abedini, A. Plesner, C. B. Verchere, D. P. Raleigh, Biochemistry (Mosc.) 2010, 49, 8127.

[20] L. M. Young, J. C. Saunders, R. A. Mahood, C. H. Revill, R. J. Foster, L.-H. Tu, D. P. Raleigh, S. E. Radford, A. E. Ashcroft, Nat. Chem. 2015, 7, 73.

[21] H. Noor, P. Cao, D. P. Raleigh, Protein Sci. Publ. Protein Soc. 2012, 21, 373.

[22] C. Zelus, A. Fox, A. Calciano, B. S. Faridian, L. A. Nogaj, D. A. Moffet, Open Biochem. J. 2012, 6, 66.

[23] R. Mishra, D. Sellin, D. Radovan, A. Gohlke, R. Winter, ChemBioChem 2009, 10, 445.

[24] L.-H. Tu, L. M. Young, A. G. Wong, A. E. Ashcroft, S. E. Radford, D. P. Raleigh, Biochemistry (Mosc.) 2014, DOI 10.1021/bi501016r.

[25] M. Daval, S. Bedrood, T. Gurlo, C.-J. Huang, S. Costes, P. C. Butler, R. Langen, Amyloid 2010, 17, 118.

[26] S. Sparks, G. Liu, K. J. Robbins, N. D. Lazo, Biochem. Biophys. Res. Commun. 2012, 422, 551.

[27] C. Adessi, C. Soto, Curr. Med. Chem. 2002, 9, 963.

[28] B. Helms, E. W. Meijer, Science 2006, 313, 929.

[29] P. K. Maiti, B. Bagchi, J. Chem. Phys. 2009, 131, 214901.

[30] C. F. Jones, R. A. Campbell, A. E. Brooks, S. Assemi, S. Tadjiki, G. Thiagarajan, C. Mulcock, A. S. Weyrich, B. D. Brooks, H. Ghandehari, D. W. Grainger, ACS Nano 2012, 6, 9900.

[31] N. K. Geitner, R. R. Powell, T. Bruce, D. A. Ladner, P. C. Ke, RSC Adv. 2013, 3, 25930.

[32] S. Svenson, D. A. Tomalia, Adv. Drug Deliv. Rev. 2005, 57, 2106.

[33] C. Yiyun, X. Tongwen, Eur. J. Med. Chem. 2005, 40, 1188.

[34] A. Asthana, A. S. Chauhan, P. V. Diwan, N. K. Jain, AAPS PharmSciTech 2005, 6, E536.

This article is protected by copyright. All rights reserved. 
[35] P. Kolhe, E. Misra, R. M. Kannan, S. Kannan, M. Lieh-Lai, Int. J. Pharm. 2003, 259, 143.

[36] R. S. DeFever, N. K. Geitner, P. Bhattacharya, F. Ding, P. C. Ke, S. Sarupria, Environ. Sci. Technol. 2015, 49, 4490.

[37] N. K. Geitner, B. Wang, R. E. Andorfer, D. A. Ladner, P. C. Ke, F. Ding, Environ. Sci. Technol. 2014, 48, 12868.

[38] O. Klementieva, E. Aso, D. Filippini, N. Benseny-Cases, M. Carmona, S. Juvés, D. Appelhans, J. Cladera, I. Ferrer, Biomacromolecules 2013, 14, 3570.

[39] S. Supattapone, H. O. Nguyen, F. E. Cohen, S. B. Prusiner, M. R. Scott, Proc. Natl. Acad. Sci. U. S. A. $1999,96,14529$.

[40] B. Klajnert, D. Appelhans, H. Komber, N. Morgner, S. Schwarz, S. Richter, B. Brutschy, M. lonov, A. K. Tonkikh, M. Bryszewska, B. Voit, Chem. Weinh. Bergstr. Ger. 2008, 14, 7030.

[41] M. Fischer, D. Appelhans, S. Schwarz, B. Klajnert, M. Bryszewska, B. Voit, M. Rogers, Biomacromolecules 2010, 11, 1314.

[42] S. Supattapone, H. Wille, L. Uyechi, J. Safar, P. Tremblay, F. C. Szoka, F. E. Cohen, S. B. Prusiner, M. R. Scott, J. Virol. 2001, 75, 3453.

[43] J. Solassol, C. Crozet, V. Perrier, J. Leclaire, F. Béranger, A.-M. Caminade, B. Meunier, D. Dormont, J.-P. Majoral, S. Lehmann, J. Gen. Virol. 2004, 85, 1791.

[44] J. M. McCarthy, D. Appelhans, J. Tatzelt, M. S. Rogers, Prion 2013, 7, 198.

[45] O. Nowacka, D. Shcharbin, B. Klajnert-Maculewicz, M. Bryszewska, Colloids Surf. B Biointerfaces 2014, 116, 757.

[46] K. Karolczak, S. Rozalska, M. Wieczorek, M. Labieniec-Watala, C. Watala, Int. J. Pharm. 2012, 436, 508.

[47] B. M. Cummin, J. Lim, E. E. Simanek, M. V. Pishko, G. L. Coté, Biomed. Opt. Express 2011, 2, 1243

[48] P. Nedumpully-Govindan, F. Ding, Sci. Rep. 2015, 5, DOI 10.1038/srep08240.

[49] R. Kodali, R. Wetzel, Curr. Opin. Struct. Biol. 2007, 17, 48.

[50] R. Kayed, J. Bernhagen, N. Greenfield, K. Sweimeh, H. Brunner, W. Voelter, A. Kapurniotu, J. Mol. Biol. 1999, 287, 781.

[51] K. C. Liu, Y. Yeo, Mol. Pharm. 2013, 10, 1695.

[52] T. Ban, D. Hamada, K. Hasegawa, H. Naiki, Y. Goto, J. Biol. Chem. 2003, 278, 16462.

[53] R. Khurana, C. Coleman, C. Ionescu-Zanetti, S. A. Carter, V. Krishna, R. K. Grover, R. Roy, S. Singh, J. Struct. Biol. 2005, 151, 229.

[54] J. J. W. Wiltzius, S. A. Sievers, M. R. Sawaya, D. Cascio, D. Popov, C. Riekel, D. Eisenberg, Protein Sci. 2008, 17, 1467.

[55] P. K. Maiti, T. Çağın, G. Wang, W. A. Goddard, Macromolecules 2004, 37, 6236.

[56] A. Hoorens, M. Van de Casteele, G. Klöppel, D. Pipeleers, J. Clin. Invest. 1996, 98, 1568.

[57] Y. Bram, A. Frydman-Marom, I. Yanai, S. Gilead, R. Shaltiel-Karyo, N. Amdursky, E. Gazit, Sci. Rep. 2014, 4, DOI 10.1038/srep04267.

[58] I. Nicoletti, G. Migliorati, M. C. Pagliacci, F. Grignani, C. Riccardi, J. Immunol. Methods 1991, $139,271$.

[59] K. Pillay, P. Govender, BioMed Res. Int. 2013, 2013, 826706.

[60] N. Feliu, P. Kohonen, J. Ji, Y. Zhang, H. L. Karlsson, L. Palmberg, A. Nyström, B. Fadeel, ACS Nano 2015, 9, 146.

[61] S. Li, C. Schöneich, R. T. Borchardt, Biotechnol. Bioeng. 1995, 48, 490.

[62] K. B. Katarzyna Winnicka, J. Health Sci. - J Health SCI 2009, 55, 169.

[63] B. Wang, S. A. Seabrook, P. Nedumpully-Govindan, P. Chen, H. Yin, L. Waddington, V. C. Epa, D. A. Winkler, J. K. Kirby, F. Ding, P. C. Ke, Phys. Chem. Chem. Phys. 2014, 17, 1728.

[64] D. C. Rapaport The Art of Molecular Dynamics Simulation, $2^{\text {nd }}$ ed.; Cambridge University Press: New York, 2004

[65] M. P. Allen, D. J. Tildesley, Computer Simulation of Liquids, Clarendon Press, 1989.

[66] F. Ding, D. Tsao, H. Nie, N. V. Dokholyan, Structure 2008, 16, 1010.

This article is protected by copyright. All rights reserved. 
[67] F. Ding, N. V. Dokholyan, PLoS Comput Biol 2006, 2, e85.

[68] D. Shirvanyants, F. Ding, D. Tsao, S. Ramachandran, N. V. Dokholyan, J. Phys. Chem. B 2012, $116,8375$.

[69] A. V. Karginov, F. Ding, P. Kota, N. V. Dokholyan, K. M. Hahn, Nat. Biotechnol. 2010, 28, 743.

[70] T. Lazaridis, M. Karplus, Curr. Opin. Struct. Biol. 2000, 10, 139.

[71] S. Yin, L. Biedermannova, J. Vondrasek, N. V. Dokholyan, J. Chem. Inf. Model. 2008, 48, 1656.

[72] R. P. R. Nanga, J. R. Brender, S. Vivekanandan, A. Ramamoorthy, Biochim. Biophys. Acta BBA Biomembr. 2011, 1808, 2337.

[73] G. S. Manning, J. Chem. Phys. 1969, 51, 924.

[74] R. Srinivasan, G. D. Rose, Proc. Natl. Acad. Sci. U. S. A. 1999, 96, 14258.

[75] F. Ding, J. J.LaRocque, N. V. Dokholyan, J. Biol. Chem. 2005, 280, 40235.

[76] M. D. McKenzie, N. L. Dudek, L. Mariana, M. M. Chong, J. A. Trapani, T. W. Kay, H. E. Thomas, Int. Immunol. 2006, 18, 837.

[77] M. D. McKenzie, E. Jamieson, E. S. Jansen, C. L. Scott, D. C. S. Huang, P. Bouillet, J. Allison, T. W. H. Kay, A. Strasser, H. E. Thomas, Diabetes 2010, 59, 644.

[78] S. Casas, A. Novials, F. Reimann, R. Gomis, F. M. Gribble, Diabetologia 2008, 51, 2252.

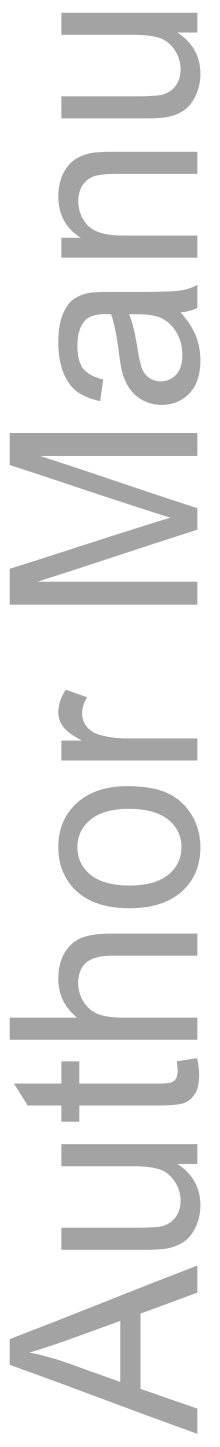

This article is protected by copyright. All rights reserved. 


\section{Figures}

A
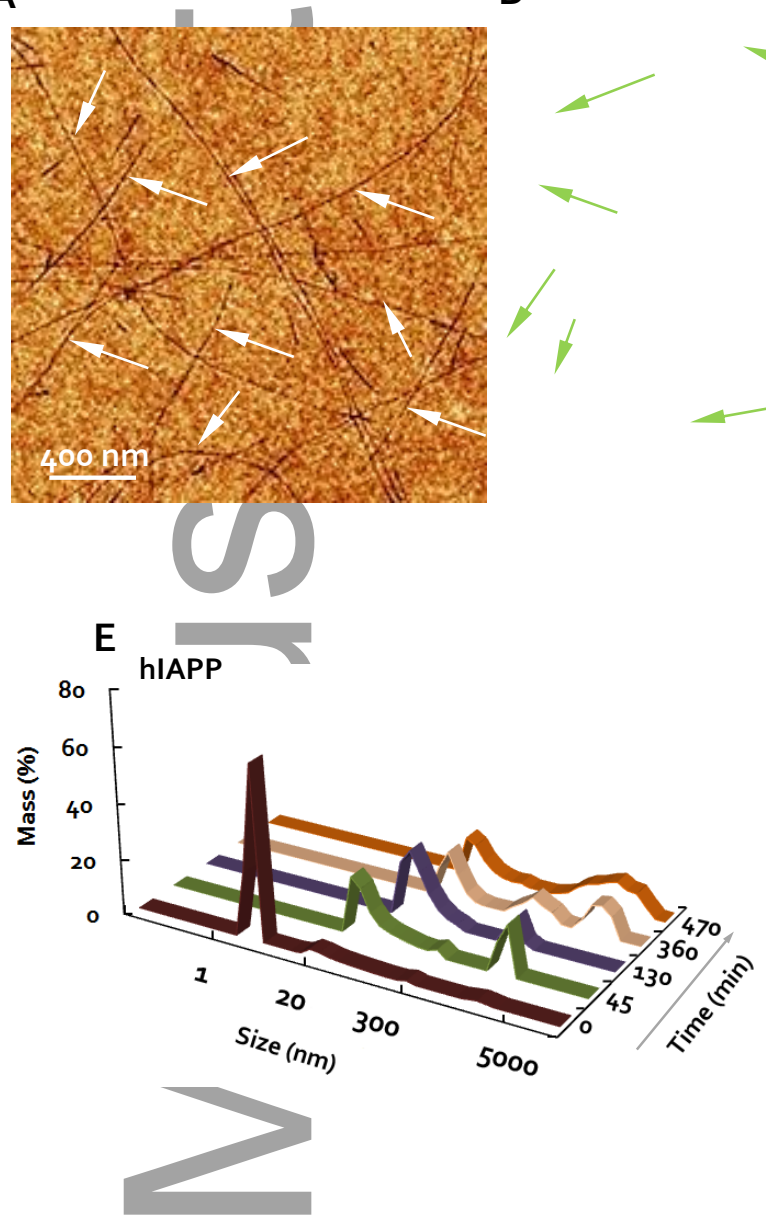

C

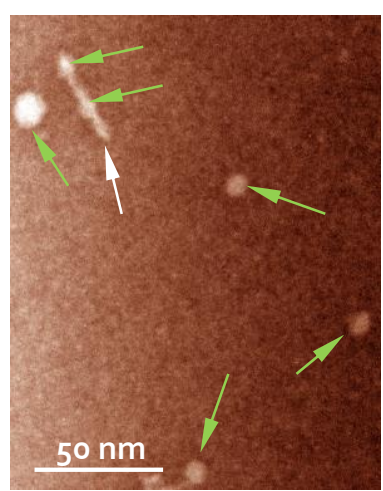

D

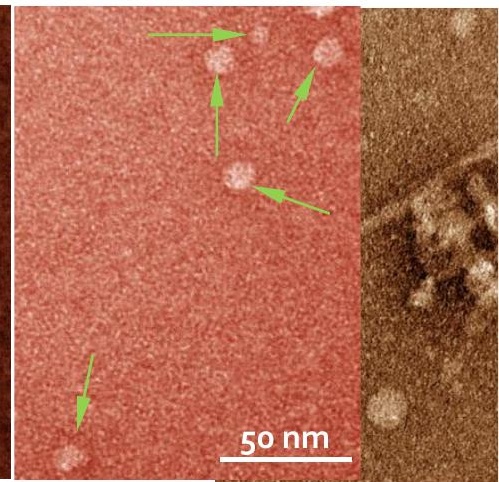

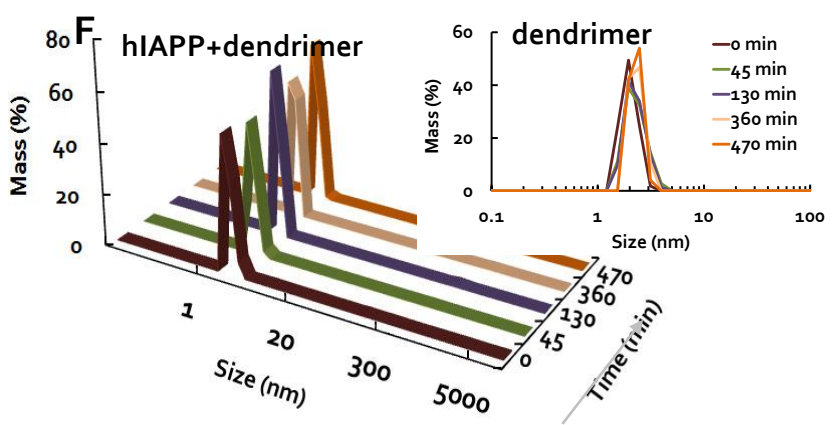

Figure 1. TEM (A-D) and high-throughput DLS (E, F) characterizations of hIAPP aggregation and its inhibition by G3 PAMAM-OH dendrimers. A: TEM image of hIAPP amyloid fibrils, mostly at $1 \mu \mathrm{m}$ or longer, indicated by white arrows. B, C: TEM image of much shortened and sparsely distributed hIAPP amyloid fibrils (exemplified by white arrows) induced by PAMAM-OH dendrimer. B-D: The spherical particles (exemplified by green arrows), a few nanomaters in size, are inferred to be hIAPP-dendrimer clusters as they were entirely absent in the dendrimer (Figure S1) or hIAPP control images (A). This is further corroborated by the co-localizations of such spherical particles with the fibrils in B and C indicating binding between the dendrimer and peptide species. Incubation times for A-D: overnight. hIAPP concentrations in A-C: $0.1 \mathrm{mg} / \mathrm{mL}(25 \mu \mathrm{M})$ and $0.05 \mathrm{mg} / \mathrm{mL}(12.5 \mu \mathrm{M})$, respectively. Dendrimer concentration in B-D: $0.16 \mathrm{mg} / \mathrm{mL}(23 \mu \mathrm{M})$. E, F: Time evolutions of the hydrodynamic diameters of hIAPP $(19 \mu \mathrm{M})$ and hIAPP-dendrimer mixture at a $6 \mu \mathrm{L}: 4 \mu \mathrm{L}$ volume ratio (or 6:7 in molar ratio), respectively. Inset of F: Size distribution of PAMAM-OH

This article is protected by copyright. All rights reserved. 
dendrimer $(33 \mu \mathrm{M})$ over time.

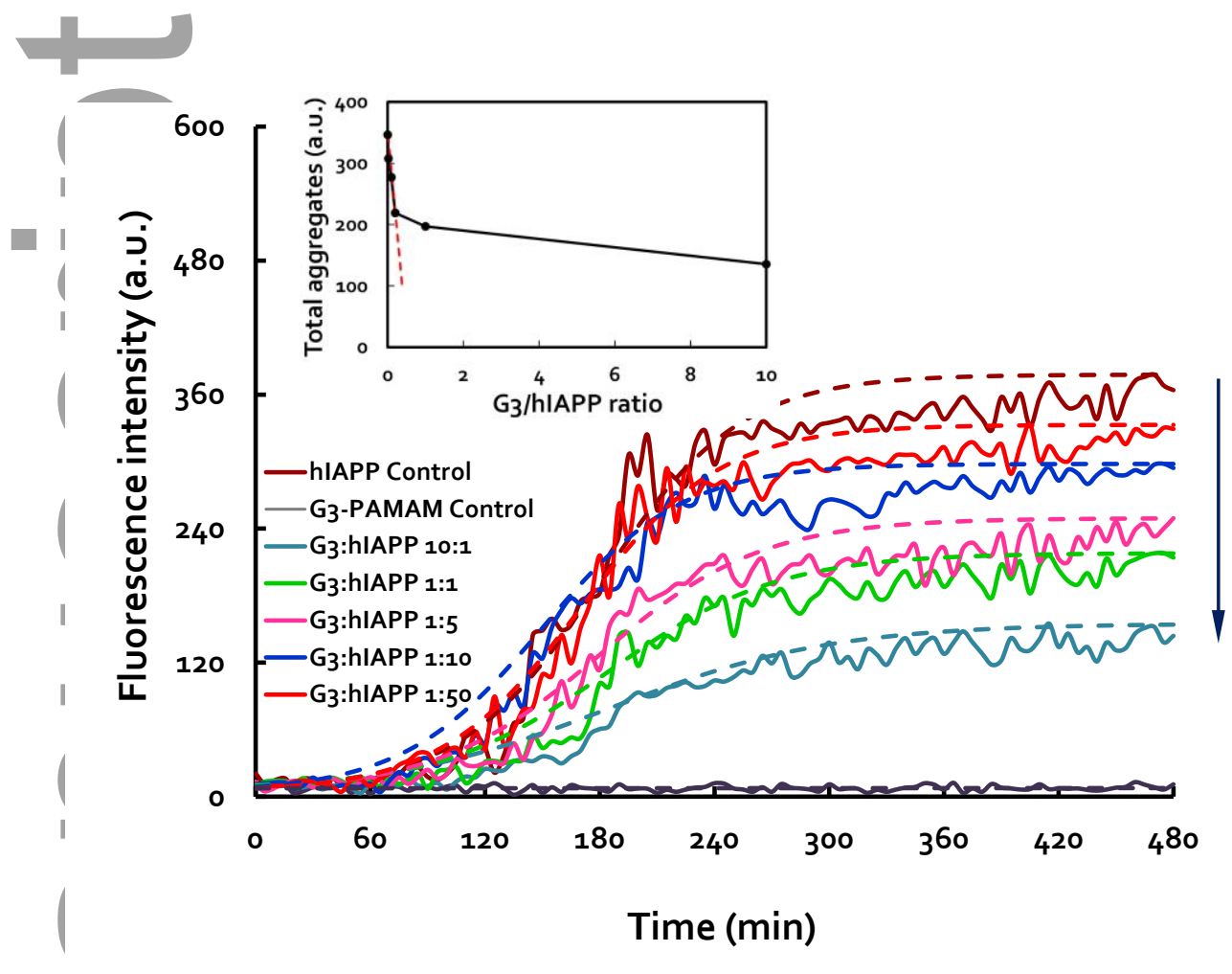

Figure 2. ThT assay showing the effect of G3 PAMAM-OH dendrimer on hIAPP aggregation. The inhibition effect of the dendrimer on hIAPP aggregation is evidenced by the monotonic decrease of the ThT fluorescence intensity with increased dendrimer concentration, indicated by the arrow to the right. The dendrimer control showed negligible fluorescence. hIAPP: $10 \mu \mathrm{M}$. PAMAM-OH dendrimer: $0.2 \sim 100 \mu \mathrm{M}$. The assay was run for $8 \mathrm{~h}$. Excitation/Emission: 440/485 nm. (Inset) Total amount of aggregates, corresponding to the maximum fluorescence intensities obtained by the sigmoidal fitting (Methods; dashed lines), is shown as a function of dendrimer/hIAPP ratio. At relatively low dendrimer/hIAPP ratios $(\leq 0.2)$, a linear decrease of the total aggregates was observed (red lashed line). Such linear decrease levelled off at higher dendrimer/hIAPP ratios.

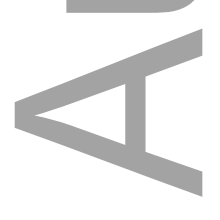

This article is protected by copyright. All rights reserved. 

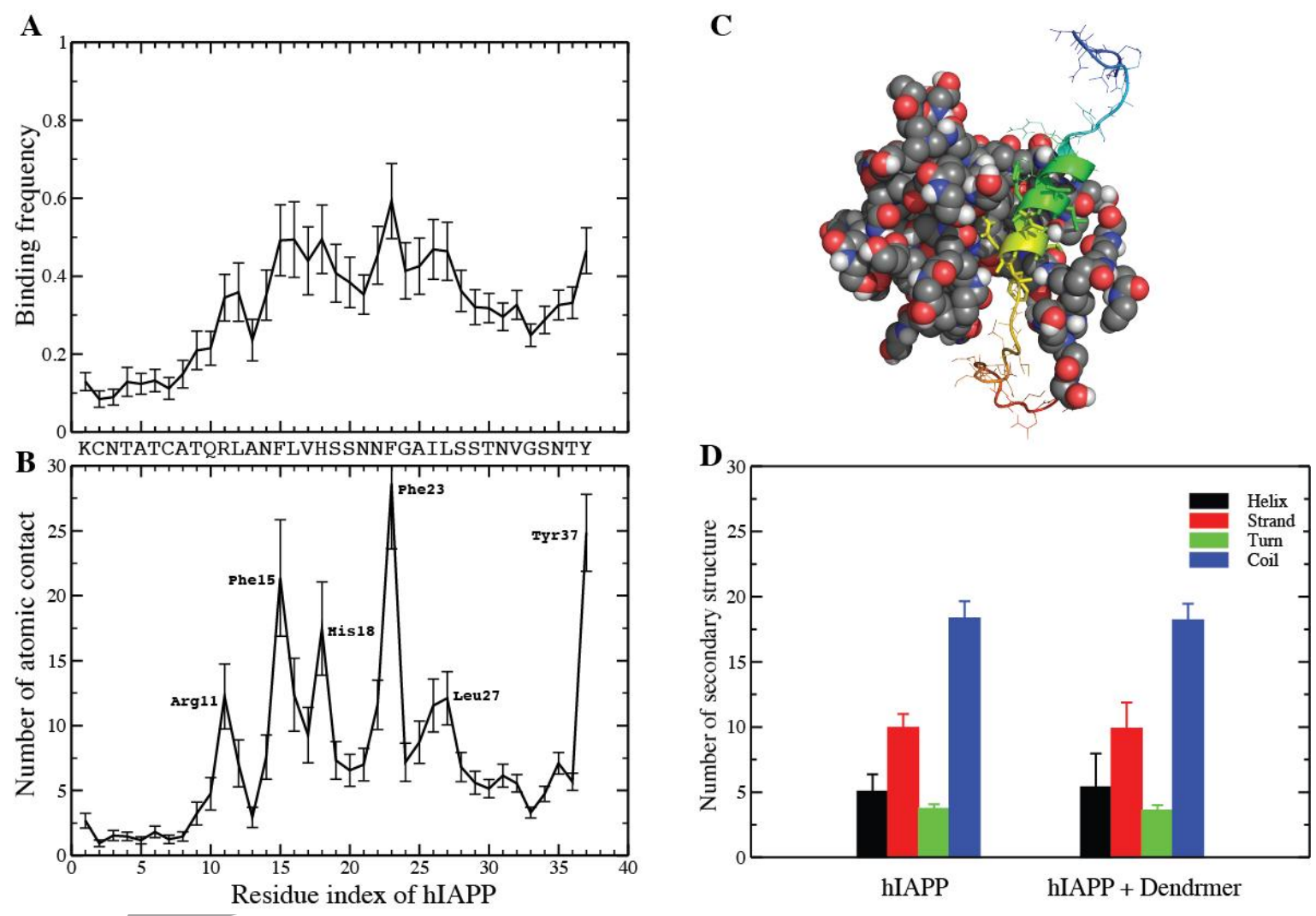

Figure 3. (A) Binding frequency and (B) number of atomic contacts between a G3 PAMAM$\mathrm{OH}$ dendrimer and individual hIAPP residues. (C) A snapshot from DMD simulations, where the dendrimer is shown in spherical representation in gray. The peptide in cartoon representation is colored rainbow, where blue corresponds to the $\mathrm{N}$-terminal and red denotes the C-terminal. (D) Secondary structure contents for simulations of hIAPP alone and in the presence of PAMAM-OH dendrimer.

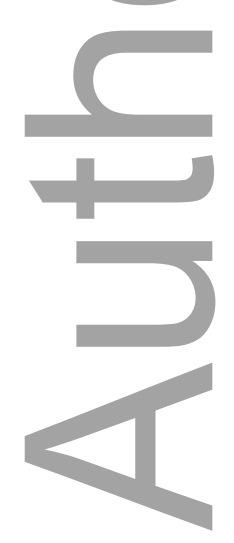

This article is protected by copyright. All rights reserved. 

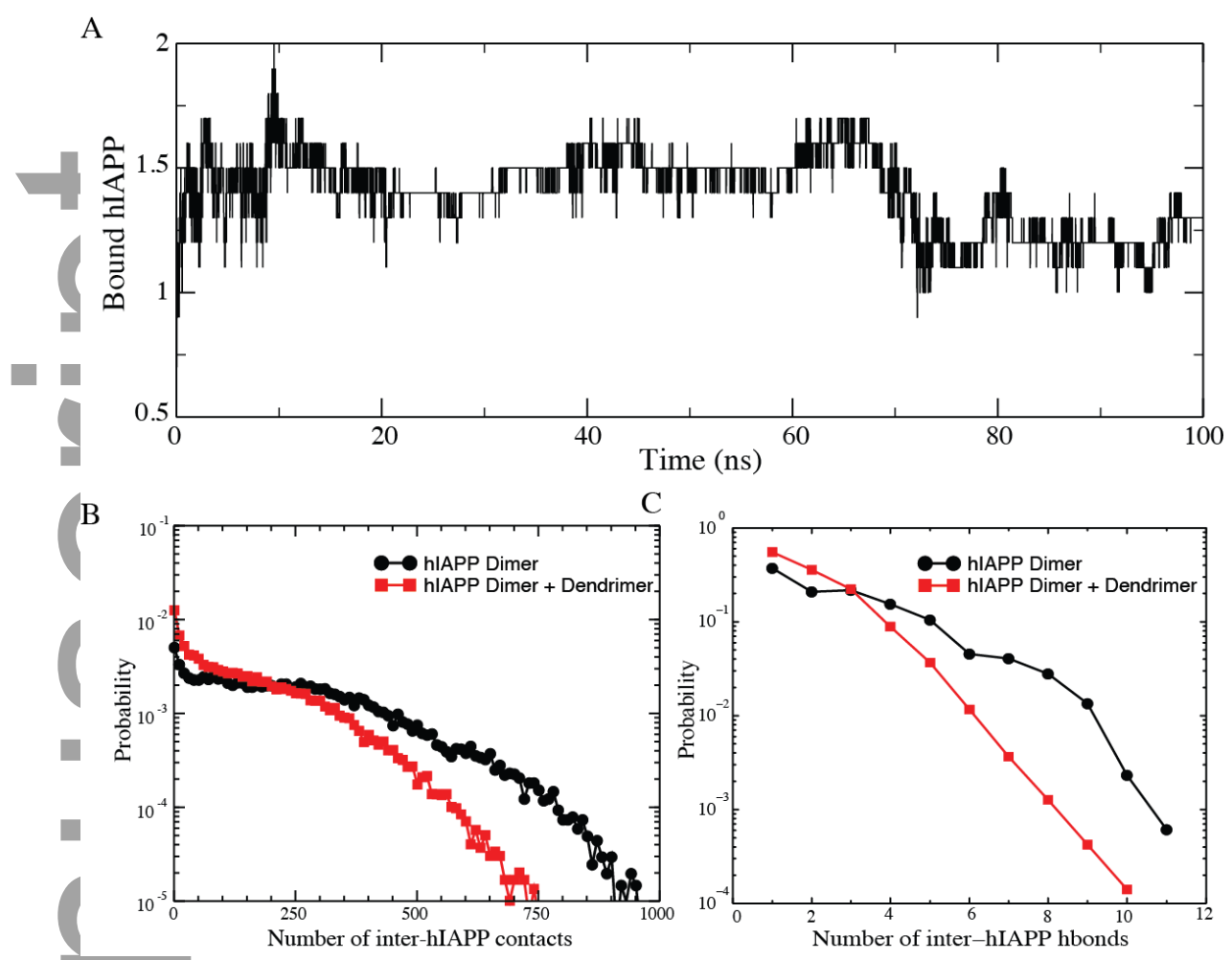

Figure 4. (A) The number of hIAPP peptides bound to a G3 PAMAM-OH dendrimer as a function of the simulation time, which was averaged over ten independent simulations (e.g., Fig. S1). (B) The probability densities of the number of inter-hIAPP contacts, $P_{n c}$ and (C) the number of inter-hIAPP hydrogen bonds, $P_{\text {hbond, }}$ for simulations of both hIAPP dimer and hIAPP dimer interacting with a single PAMAM-OH dendrimer.

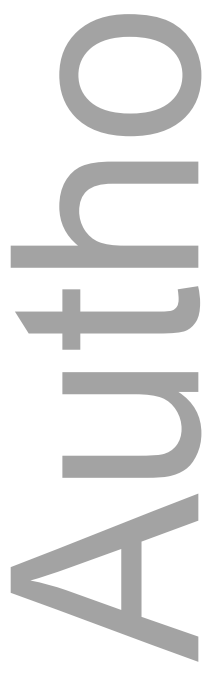

This article is protected by copyright. All rights reserved. 
A

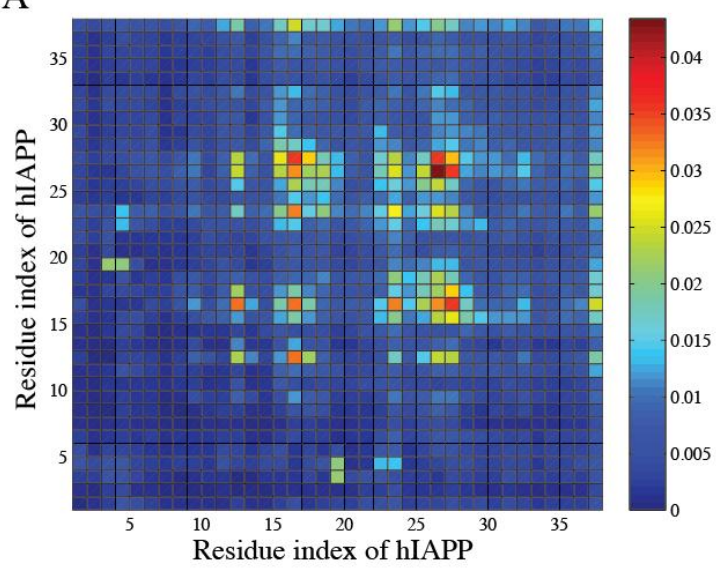

$\mathrm{B}$

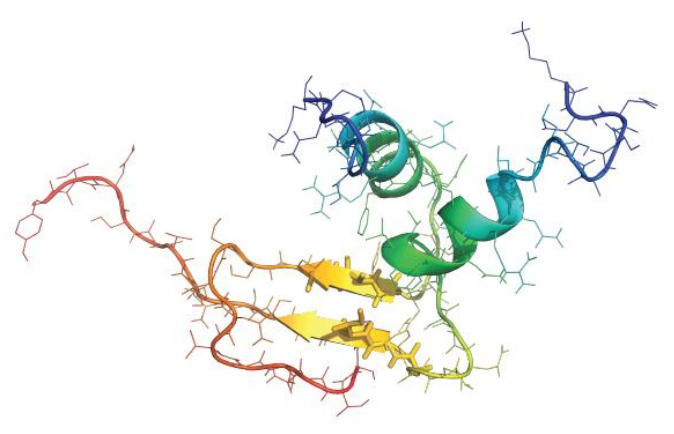

$\mathrm{C}$

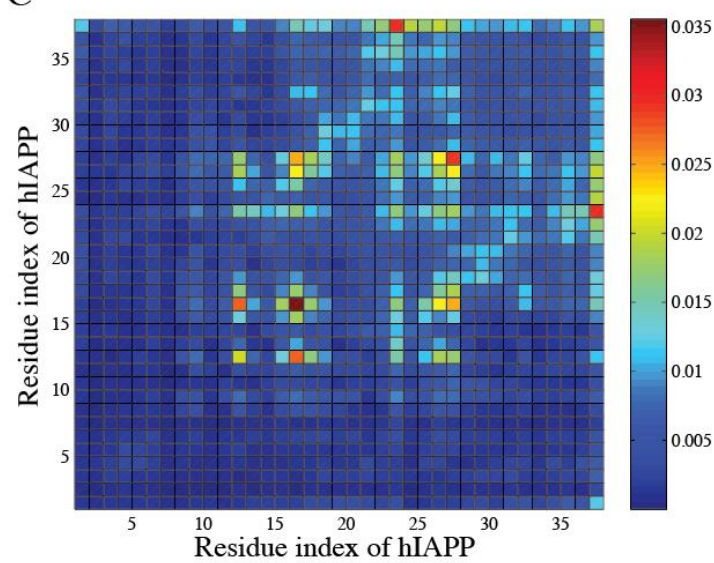

$\mathrm{D}$

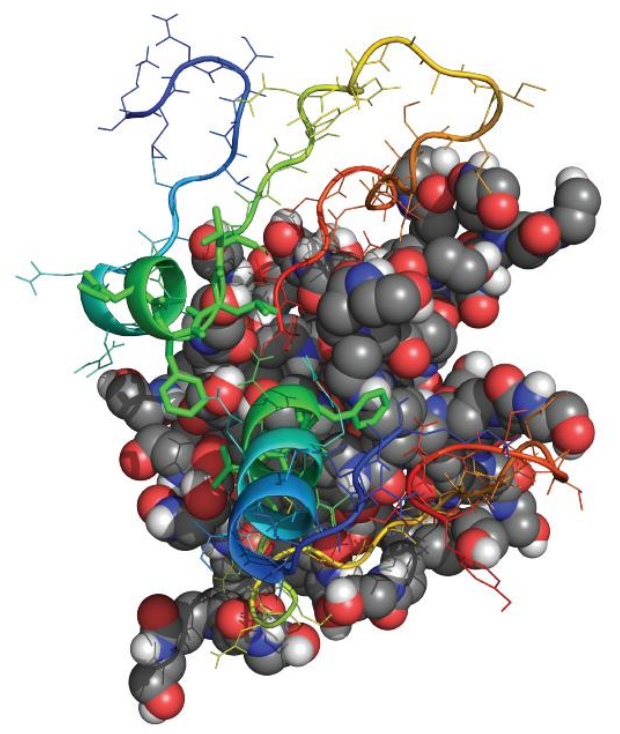

Figure 5. Dimerization simulations of hIAPP with and without a G3 PAMAM-OH dendrimer. The residue-wise contact frequencies between two hIAPP peptides were obtained from DMD simulations of two hIAPP monomers alone $(A)$ and interacting with a PAMMA-OH dendrimer $(C)$. We note the difference in the scale of color bars, where the hIAPPs alone case show a higher binding frequency. For the hIAPP dimer alone, the interactions between the two peptides are mostly between the amyloidogenic regions (residues 22-29) of each chain. In the presence of the PAMAM-OH dendrimer, the interactions are more scattered. (B) A typical snapshot structure from the hIAPP dimer simulation. The peptide in cartoon representation is colored rainbow, where blue corresponds to the $\mathrm{N}$-terminal and red denotes the C-terminal. A beta-sheet is formed between the amyloidogenic regions (the yellow regions), while the $\mathrm{N}$-termini remain helical. (D) A snapshot structure from the hIAPP dimer simulation in the presence of the PAMAM-OH dendrimer (in spherical representation). The amyloidogentic region of one peptide was buried by binding with the dendrimer. The hydrophobic region around residues $15-17\left(F^{15} L V^{17}\right)$, which formed interpeptide interactions, was highlighted in sticks. 

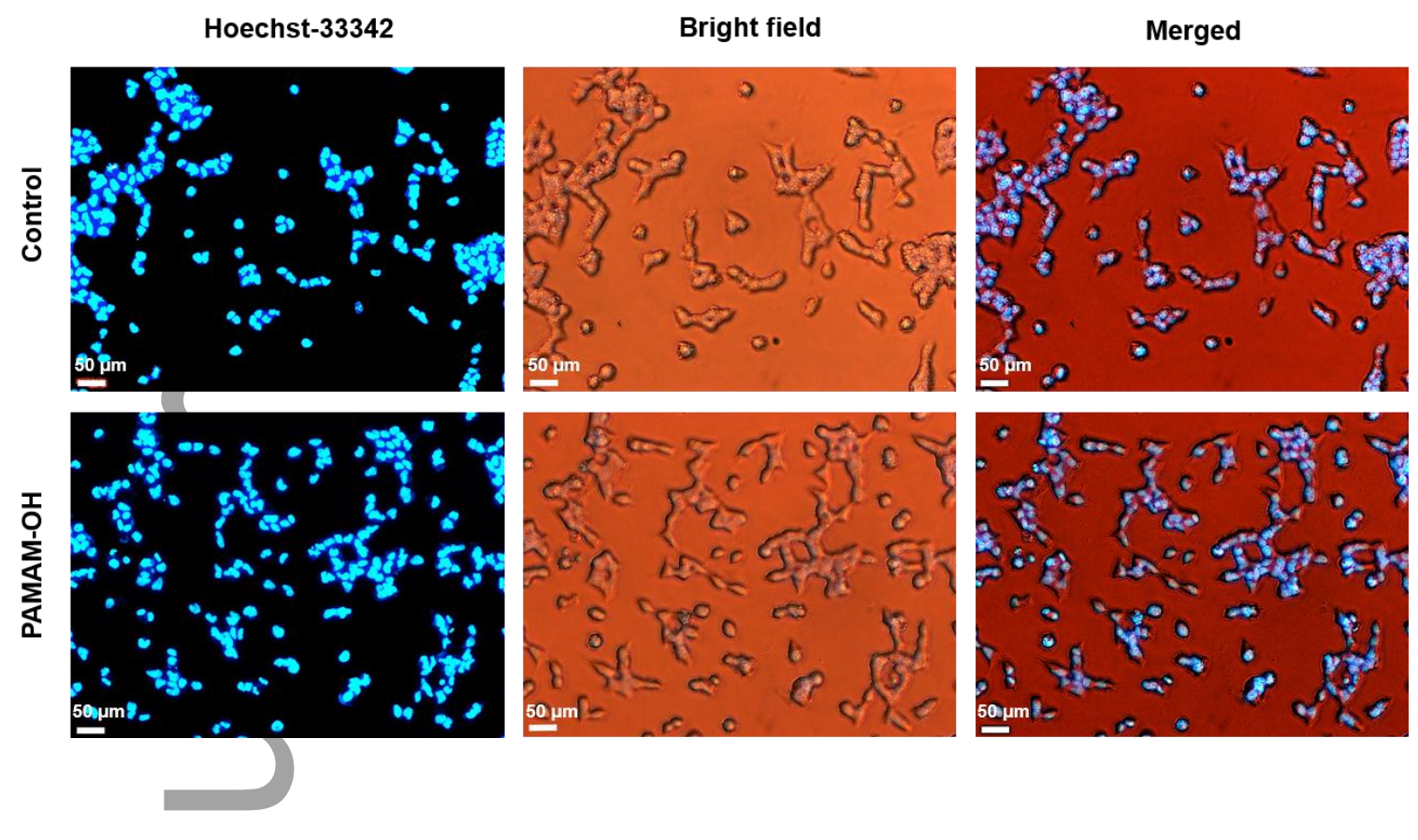

Figure 6. $7.5 \times 104$ NIT-1 cells were treated with PAMAM-OH $(6 \mu \mathrm{M})$ for $24 \mathrm{~h}$ and nuclear DNA staining evaluated by Hoechst-33342 (blue) and cell morphology by light microscopy. The blue staining corresponds to nuclear DNA in the cells, indicating that PAMAM-OH did not interfere in the viability assay of Figure 7. Images are representative of 5 different fields taken for each sample, Scale bars: $50 \mu \mathrm{m}$.

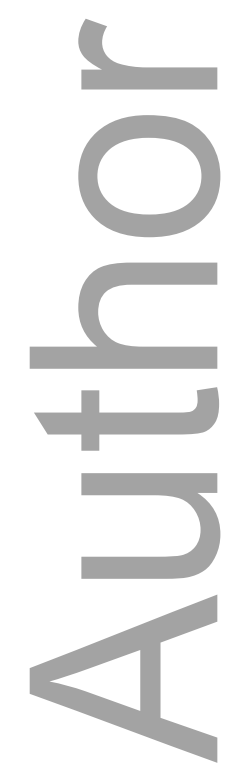

This article is protected by copyright. All rights reserved. 


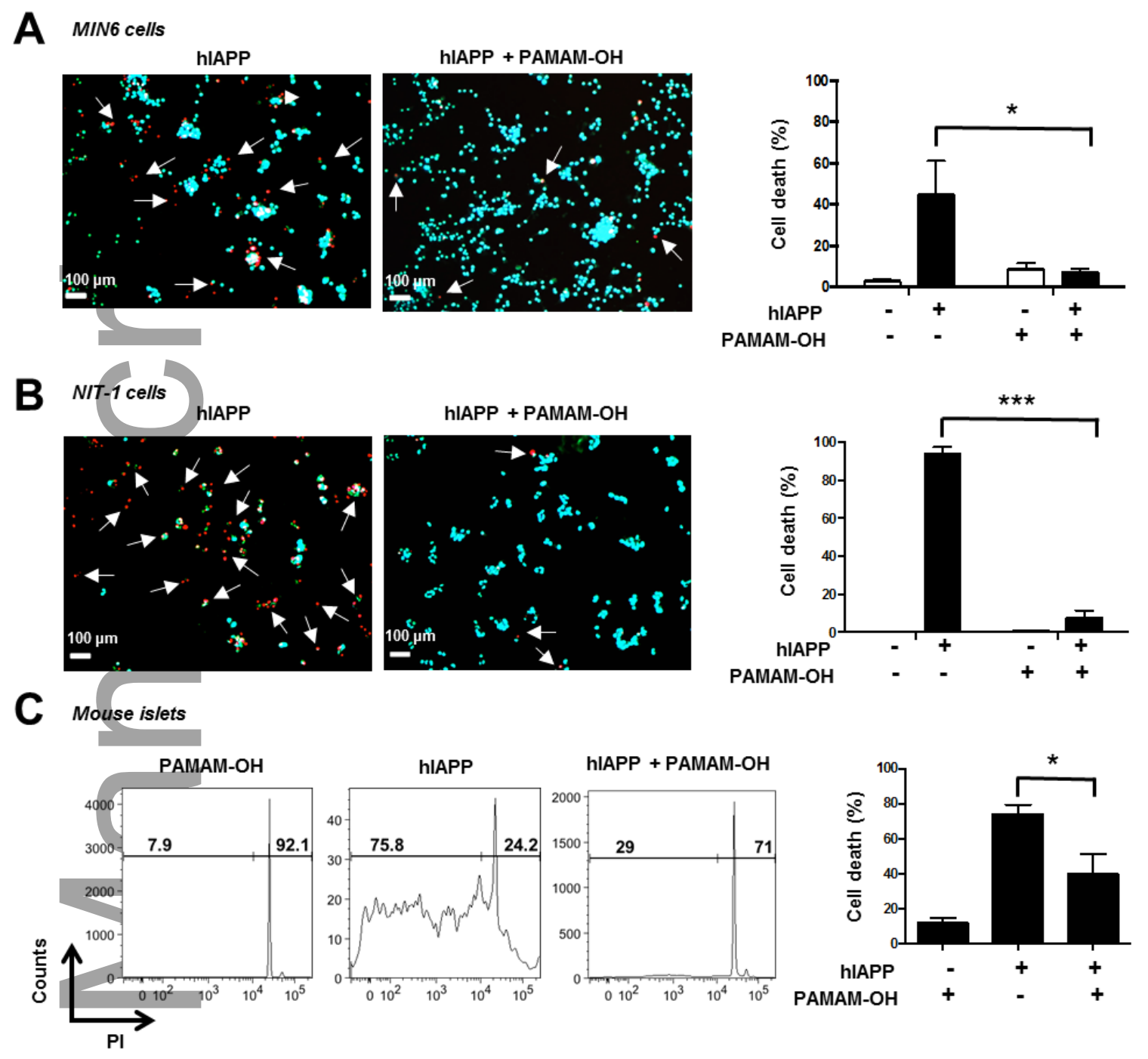

Figure 7. G3 PAMAM-OH dendrimer protects $\beta$-cells from hIAPP-induced cell death. (A) MIN-6 cells were left untreated (control) or incubated with PAMAM-OH, hIAPP or combination for $24 \mathrm{~h}$. Cell death was evaluated by Hoechst-33342 (blue)/propidium iodide (red). White arrows indicate propidium iodide positive cells. Data shown are means \pm SEM of 3 independent experiments. ${ }^{*} P<0.05$. Scale bar: $100 \mu \mathrm{m}$. (B) NIT-1 cells were untreated (control) or incubated with PAMAM-OH, hIAPP or combination for $24 \mathrm{~h}$. Cell death was evaluated by Hoechst-33342 (blue)/propidium iodide (red). White arrows indicate propidium iodide positive cells. Data shown are means \pm SEM of 3 independent experiments. ${ }^{* * *} P<0.001$. Scale bar: $100 \mu \mathrm{m}$. (C) DNA fragmentation was measured by flow cytometry in islets from C57BL/6 mice cultured in medium containing PAMAM-OH, hIAPP, or combination for $48 \mathrm{~h}(\mathrm{n}=4)$. Data shown are means \pm SEM, $* P<0.05$. 

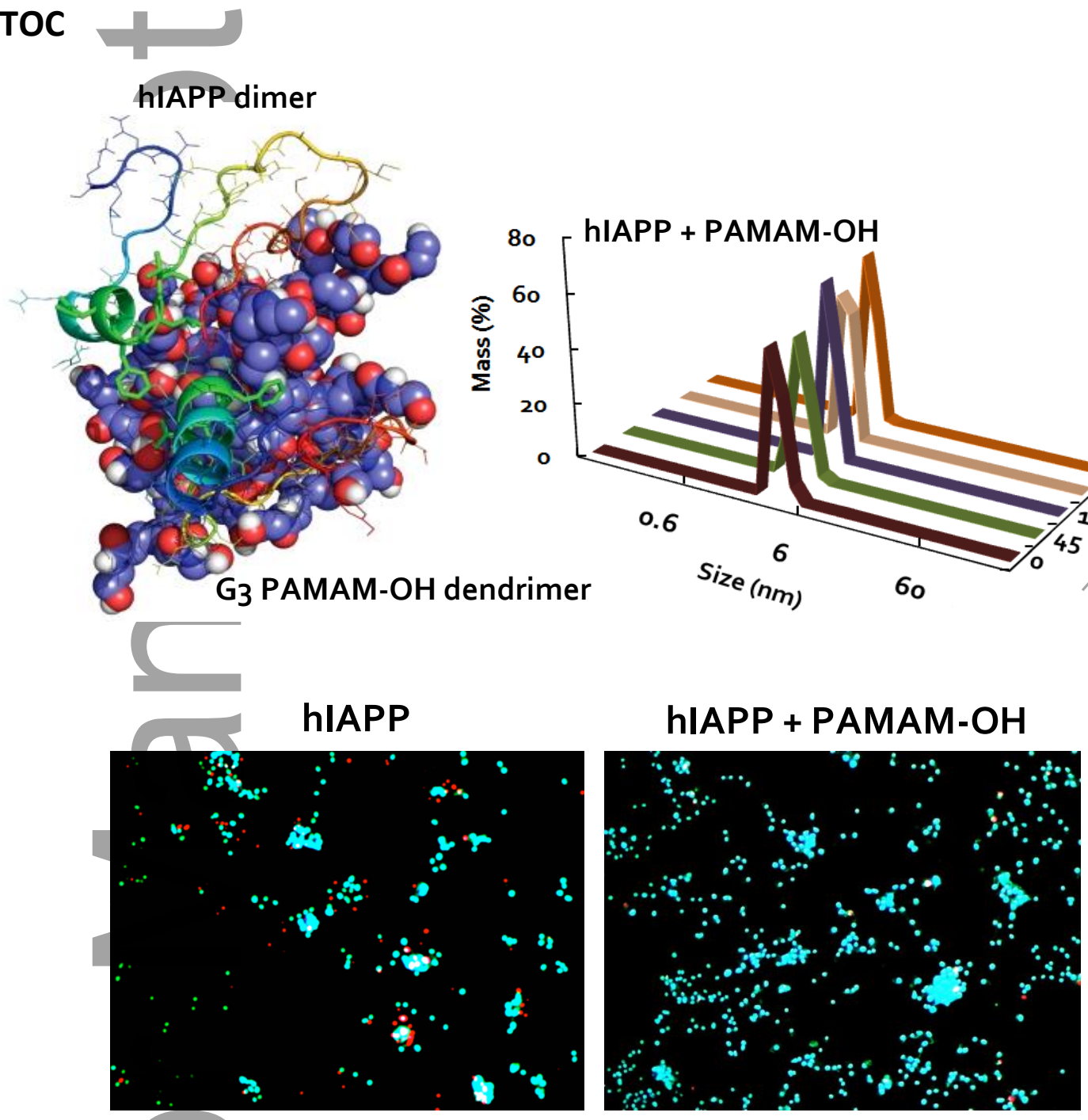

Inhibition of hIAPP aggregation and cytotoxicity by G3 PAMAM-OH dendrimer. (Top

left) DMD simulation illustrating that binding with a G3 PAMAM-OH dendrimer (ball representation) inhibits the self-association of the amyloidogenic sequences (in yellow) between two hIAPP peptides (ribbon representation). (Top right) High-throughput DLS showing inhibited hIAPP aggregation over time. (Low left) Pronounced hIAPP toxicity in MIN6 cells. (Low right) Minimal hIAPP toxicity in MIN6 cells in the presence of G3 PAMAM-OH dendrimers. 


\section{University Library}

\section{- M M N E R VA A gateway to Melbourne's research publications}

Minerva Access is the Institutional Repository of The University of Melbourne

\section{Author/s:}

Gurzov, EN;Wang, B;Pilkington, EH;Chen, P;Kakinen, A;Stanley, WJ;Litwak, SA;Hanssen, EG;Davis, TP;Ding, F;Ke, PC

Title:

Inhibition of hIAPP Amyloid Aggregation and Pancreatic beta-Cell Toxicity by $\mathrm{OH}$-Terminated PAMAM Dendrimer

Date:

2016-03-23

Citation:

Gurzov, E. N., Wang, B., Pilkington, E. H., Chen, P., Kakinen, A., Stanley, W. J., Litwak, S. A., Hanssen, E. G., Davis, T. P., Ding, F. \& Ke, P. C. (2016). Inhibition of hIAPP Amyloid Aggregation and Pancreatic beta-Cell Toxicity by $\mathrm{OH}$-Terminated PAMAM Dendrimer. SMALL, 12 (12), pp.1615-1626. https://doi.org/10.1002/smll.201502317.

Persistent Link:

http://hdl.handle.net/11343/290861 